\title{
Pre-Emptive oral Pregabalin for Pain Relief After Laparoscopic Cholecystectomy
}

\author{
Dr. Ranu Surana ${ }^{1}$,Dr. Shashikala Chaturvedi ${ }^{2}$,Dr. Durga Jethava ${ }^{3}$ \\ ${ }^{1} 3 r d$ Year Post graduate, ${ }^{2}$ Professor, ${ }^{3}$ Professor \& Head \\ Dept of Anesthesiology, Mahatma Gandhi University of Medical Sciences and Technology, Jaipur, India
}

\begin{abstract}
:
Introduction: Aim of the present study was to evaluate the role of pre-emptive analgesia with oral pregabalin in patients undergoing laparoscopic cholecystectomy

Methods: The study enrolled 60 adult patients (16-60 yr) undergoing laparoscopic cholecystectomy. Patients were randomly assigned into two groups of 30 each to receive either a matching placebo or pregabalin $150 \mathrm{mg}$, $1 \mathrm{~h}$ before induction of anaesthesia. Assessment of pain at rest (static) and during coughing (dynamic) were done by a 10 point visual analogue scale (VAS) at 15, 30, 45, 60, 90, 120 mins and 6, 12 and 24 hours after arrival in the recovery. Primary outcomes were severity of postoperative pain and tramadol requirement. Side effects including nausea, vomiting, sedation and dizziness were also recorded.

Results: 23 males and 37 females were enrolled in the study. Both static and dynamic pain scores measured by $V A S$ were significantly less in patients receiving pregabalin compared to placebo $(p<0.01)$. Prebabalin treated patients required less rescue analgesia although this was not statistically significant $(p=0.054)$. There were no differences in side effects between the two groups.

Conclusion: Perioperative 150mg oral pregabalin in patients undergoing laparoscopic cholecystectomy is an effective and safe method of analgesia with a low incidence of adverse effects.
\end{abstract}

Keywords: Laparoscopic cholecystectomy, pain, pre-emptive analgesia, pregabalin, Visual analog scale.

\section{Introduction}

Laparoscopic cholecystectomy (LC) in itself is associated with fewer postoperative complications and less pain compared to open cholecystectomy. [1] The pain is nevertheless a major clinical problem during the early recovery in the hospital with 10-50 \% of patients experiencing persistent pain. Two to ten per cent suffer from severe chronic pain. Pain after Laparoscopic cholecystectomy is assessed with the same scales as postsurgical pain in general: the Visual Analogue Scale (VAS) and the Verbal Rating Scale (VRS). [2] When using the VAS, the patient is asked to rate his or her level of pain on a continuum between no pain (0) and the worst imaginable pain (10) by marking it on a plain 10-cm line with only the anchor points 0 and 10 at the opposite ends. Laparoscopic cholecystectomy gives rise to three sorts of pain: incisional (parietal), deep abdominal (visceral), and shoulder pain (referred visceral). Pain is most effectively controlled by combining several analgesics, each of which targets processes in the sensory pathways (transduction, transmission, modulation, and perception). Gabapentinoids are a group of anticonvulsant drugs. The two major gabapentinoids are pregabalin, and its predecessor gabapentin. They are structural analogues of gamma-amino butyric acid (GABA), but do not act on GABA receptors. Instead, they bind to alpha-2-delta subunits in voltagegated calcium channels, and reduce calcium influx into presynaptic neurons. As a consequence, the release of several excitatory neurotransmitters (glutamate, noradrenaline, and substance $\mathrm{P}$ ) is reduced, which is believed to be the mechanism behind their analgesic effects. [3]

Pregabalin has many advantages over gabapentin. Unlike gabapentin, pregabalin has highly predictable and linear pharmacokinetics. It is absorbed proportional to dose, and has high bioavailability. Maximal plasma concentration is achieved rapidly (after one hour). These properties make pregabalin attractive in clinical practice. [4] Several studies have showed that gabapentin can be used safely and efficiently for postoperative pain after various types of surgery (including LC) [5]. The results concerning pregabalin are yet inconsistent. This study was done to evaluate the role of preoperative single dose of pregabalin for attenuating postoperative pain in patients undergoing laparoscopic cholecystectomy.

\section{Materials And Methods}

This prospective, randomized, double-blind, and placebo controlled clinical study was conducted in Department of Anesthesiology at Mahatma Gandhi Medical College, Jaipur, India. The study protocol was approved from the institutional ethical committee and written informed consent was obtained from all the patients. The use of visual analogue scale (VAS) was explained to the patients during pre-anaesthesia consultation. 


\subsection{Inclusion Criteria}

The study was designed to include 60 adult patients (16-60 yr) of either sex, ASA physical status I or II, undergoing laparoscopic cholecystectomy under general anaesthesia.

\subsection{Exclusion Criteria}

1. Patients with impaired kidney or liver functions

2. History of drug or alcohol abuse

3. History of chronic pain or daily intake of analgesics

4. Uncontrolled medical disease (diabetes mellitus and hypertension)

5. History of intake of nonsteroidal anti-inflammatory drugs within $24 \mathrm{~h}$ before surgery

A sample size of twenty five by group was calculated to detect a significant difference of 2 points in Visual Analog Scale with power of $80 \%$ and significance level of $5 \%$. To take care of drop outs we enrolled thirty patients in each group. Patients meeting the inclusion criteria during the preanaesthetic evaluation were randomly assigned into two groups of 30 each with the help of a computer-generated table of random numbers, to receive either a matching placebo or pregabalin $150 \mathrm{mg}$. All the medications provided were identical, and were administered orally, $1 \mathrm{~h}$ before the induction of anaesthesia with sips of water by a staff nurse who was not involved in the study.

Anaesthesia technique was standardized in all the groups. Patients were induced with fentanyl $2 \mathrm{mcg} / \mathrm{kg}$ and propofol $2 \mathrm{mg} / \mathrm{kg}$; orotracheal intubation was facilitated by vecuronium $0.08 \mathrm{mg} / \mathrm{kg}$. Anaesthesia was maintained with inhalational isoflurane and $50 \%$ nitrous oxide in oxygen. At the end of surgery, residual neuromuscular paralysis was antagonized with neostigmine $0.08 \mathrm{mg} / \mathrm{kg}$ and glycopyrrolate $0.02 \mathrm{mg} / \mathrm{kg}$. After satisfactory recovery, the patients were extubated and shifted to the post-anaesthesia care unit.

Primary outcomes were severity of postoperative pain and postoperative tramadol requirement. Both these outcomes were assessed by an independent anaesthesia resident blinded to group allocation. Assessment of pain both at rest (static) and during coughing (dynamic) were done by a 10 point visual analogue scale (VAS) on arrival of patient to the PACU (0) and then at 15, 30, 45, 60, 90 and 120 mins and after 6, 12 and 24 hours. The following data on side effects were recorded: postoperative nausea, vomiting, sedation and dizziness. Rescue analgesia timing and dosage were also recorded. Rescue analgesia was administered on patients' request. $100 \mathrm{mg}$ of i.v. tramadol was administered and timing noted. Additional doses were also given if patient so demanded.

\subsection{Statistical Analysis}

Patient characteristic data were analysed with two-way ANOVA for continuous variables and chisquare test for categorical variables. Independent Samples t test was used to study side effects of drugs and post operative analgesic requirement in the two groups. The package SPSS 14.0 (SPSS Inc., Chicago, IL, USA) was used for statistical analysis. $\mathrm{P}<0.05$ was considered significant.

\section{Observations And Result}

From January 2015 to June 2016, a total of 60 patients undergoing Laparoscopic cholecystectomy consented and were randomized for the study. Patients were age and sex matched and also had no significant differences in vital parameters or ASA status. (Table 1)

Table 1: Patient characteristic data presented as mean (range) or as numbers.

\begin{tabular}{|c|c|c|c|}
\hline & Pregabalin group & Placebo group & Sig (p value) \\
\hline Age (years) & $42.2(25-60)$ & $41.1(24-60)$ & 0.737 \\
\hline Sex (M/F) & $11 / 19$ & $12 / 18$ & 0.795 \\
\hline Heart Rate (bpm) & $73.87(56$ to 90$)$ & $73.9(60$ to 92$)$ & 0.987 \\
\hline Blood Pressure (SBP/DBP) & $119.47 / 78.80$ & $120.67 / 79.47$ & $0.723 / 0.763$ \\
\hline ASA status (1/2) & $22 / 8$ & $25 / 5$ & 0.562 \\
\hline
\end{tabular}

\subsection{Pain Scores}

Static Pain (SP) scores measured at all the intervals in the first 24 hours after the operation were significantly lower in patients receiving placebo compared to age and sex matched controls. (Table2, Fig 1) 
Table 2: VAS Scores Static Pain

\begin{tabular}{|c|c|c|c|c|c|c|}
\hline & Pregabalin & SD & Placebo & SD & t & Sig (p value) \\
\hline SP15 & 4.4 & 1.221 & 6.2 & 1.448 & -5.206 & $<0.01$ \\
\hline SP30 & 3.8 & 0.997 & 5.4 & 0.814 & -6.812 & $<0.01$ \\
\hline SP45 & 3.6 & 0.814 & 5 & 0.91 & -6.283 & $<0.01$ \\
\hline SP60 & 3 & 0.525 & 4.73 & 0.583 & -12.095 & $<0.01$ \\
\hline SP120 & 2.47 & 0.819 & 4.13 & 0.629 & -8.839 & $<0.01$ \\
\hline SP6 & 1.93 & 0.691 & 3.47 & 0.73 & -8.351 & $<0.01$ \\
\hline SP12 & 1.07 & 0.944 & 2.27 & 0.944 & -4.921 & $<0.01$ \\
\hline SP24 & 0.8 & 0.664 & 1.93 & 0.691 & -6.473 & $<0.01$ \\
\hline
\end{tabular}

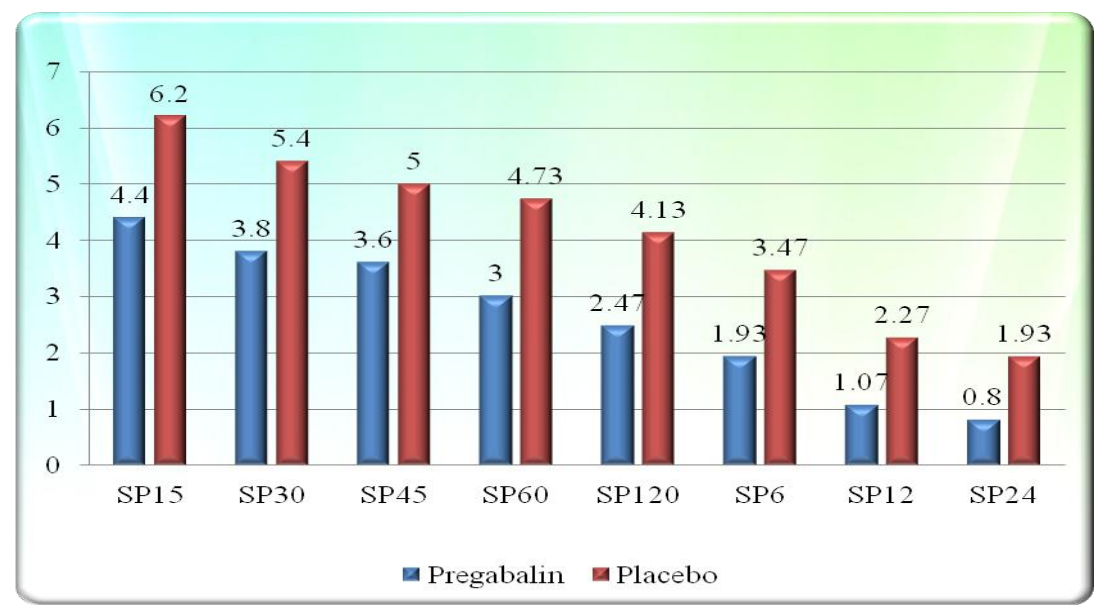

Figure 1: VAS Scores Static Pain

Similar to static pain scores, Dynamic pain (DP) measured by the VAS score after coughing was significantly less in patients recieving pregabalin $150 \mathrm{mg}$ preoperatively compared to controls. (Table 3 , Fig 2) This difference was consistent across all intervals at which pain was measured.

Table 3: VAS Scores Dynamic Pain

\begin{tabular}{|c|c|c|c|c|c|c|}
\hline & Pregabalin & SD & Placebo & SD & T & Sig (p value) \\
\hline DP15 & 5.93 & 0.944 & 7.87 & 1.224 & -6.848 & $<0.01$ \\
\hline DP30 & 5.13 & 0.819 & 7.27 & 0.785 & -10.298 & $<0.01$ \\
\hline DP45 & 4.87 & 1.042 & 6.67 & 0.884 & -7.216 & $<0.01$ \\
\hline DP60 & 4 & 0.743 & 6.33 & 0.711 & -12.428 & $<0.01$ \\
\hline DP120 & 3.87 & 0.973 & 5.4 & 0.621 & -7.273 & $<0.01$ \\
\hline DP6 & 2.93 & 0.868 & 4.4 & 0.894 & -6.444 & $<0.01$ \\
\hline DP12 & 2.47 & 1.042 & 3.27 & 0.944 & -3.116 & $<0.01$ \\
\hline DP24 & 1.67 & 0.802 & 3 & 1.05 & -5.525 & $<0.01$ \\
\hline
\end{tabular}

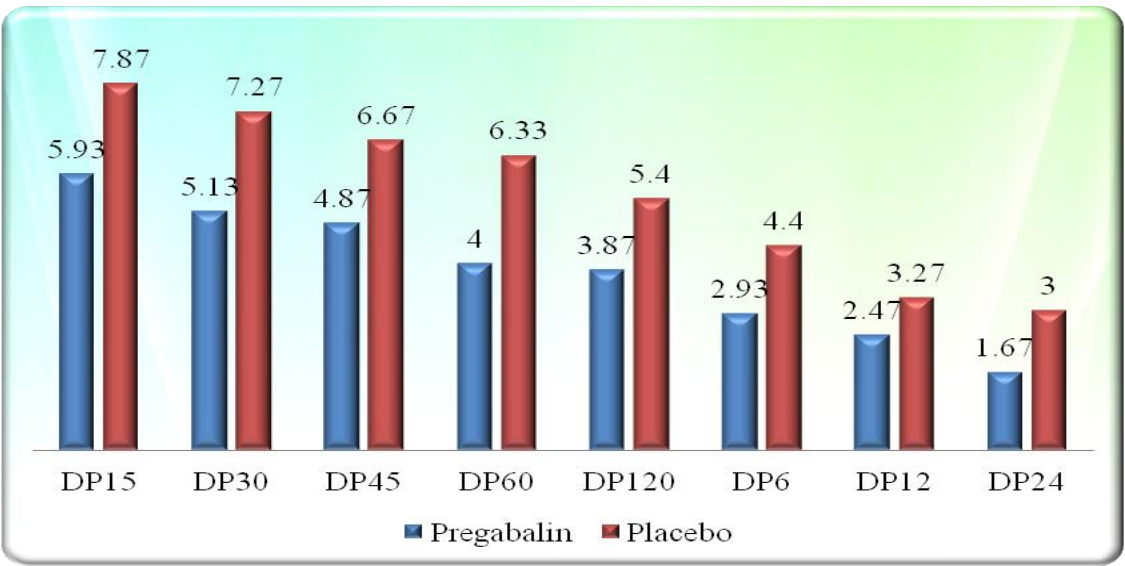

Figure 2: VAS Scores Dynamic Pain 


\subsection{Rescue Analgesic Requirement}

3 patients out of 30 in the pregabalin group needed rescue analgesia compared to 9 patients in the placebo group. (Table 4) The difference was not statistically significant ( $\mathrm{p}=0.054$ by Independent Samples $\mathrm{t}$ test).

Table 4: Analgesic Requirement

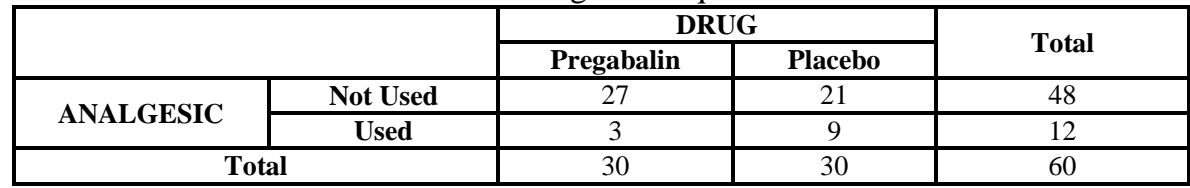

\subsection{Side Effects}

Nausea, vomiting, sedation and dizziness were recorded. There was no significant difference in incidence of side effects between the two groups. (Table 5)

Table 5: Number of patients experiencing Side Effects

\begin{tabular}{|c|c|c|c|}
\hline & Pregabalin & Placebo & p value \\
\hline Nausea & $9 / 30$ & $9 / 30$ & 1.0 \\
\hline Vomiting & $3 / 30$ & $3 / 30$ & 1.0 \\
\hline Sedation & $5 / 30$ & $4 / 30$ & 0.723 \\
\hline Dizziness & $2 / 30$ & $3 / 30$ & 0.647 \\
\hline
\end{tabular}

\section{Discussion}

The safety and effectiveness of different pharmacological interventions such as NSAIDs, opioids, and anticonvulsant analgesics in people undergoing laparoscopic cholecystectomy has been evaluated in multiple studies. The choice of such an agent is guided by factors such as efficacy, convenience of administration, costeffectiveness and safety profile.

\subsection{Demographic Profile}

In our study population, $62 \%$ of patients were females. The average age of patients included in our study was 41.6 years. There were no significant differences in age or sex distribution between the two groups. This is important because it has implications in the difference of perception of pain. $78 \%$ of our patients were in ASA grade I and the rest $22 \%$ were in ASA grade II. We did not include patients at high anesthetic risk as it could have made the post-operative course difficult to manage and hamper assessment of pain for the study.

\subsection{Difference In Pain Scores}

Pain scores (both static and dynamic) in the first 2 hours after laparoscopic cholcystectomy were reduced by 1.5 to $2 \mathrm{~cm}$ in the pregabalin group compared to placebo. The VAS scores in the 6 to 24 hour period after the operation were less by 1 to $1.5 \mathrm{~cm}$ in the pregabalin arm.

Gurusamy K in a 2014 Cochrane meta-analysis [6] studied the various pharmacological interventions for prevention or treatment of postoperative pain in people undergoing laparoscopic cholecystectomy. The authors found that the pain at 4 to 8 hours was generally reduced by about 1 to $2 \mathrm{~cm}$ on the visual analogue scale of 1 to $10 \mathrm{~cm}$ in the comparisons involving the different pharmacological agents and inactive controls. The pain at 9 to 24 hours was generally reduced by about $0.5 \mathrm{~cm}$ (a modest reduction).

\subsection{Pain}

Assessment of the intensity of acute pain at rest after surgery is important for making the patient comfortable in bed. However, adequate relief of dynamic pain during mobilization, deep breathing, and coughing is more important for reducing risks of cardiopulmonary and thromboembolic complications after surgery. In our study, we observed that preoperative single-dose pregabalin $(150 \mathrm{mg})$ was effective in reducing both the static and the dynamic components of postoperative pain. Findings similar to our study have been previously reported in few earlier studies. Agarwal et al in 2008 [7] reported reported a statistically significant decrease in pain intensity (static and dynamic) with pregabalin $150 \mathrm{mg}$. Similarly, Balaban et al [8] showed decrease in pain scores with pregabalin 150 and $300 \mathrm{mg}$ compared to placebo. Sarakatsianou in 2013 [9] demonstrated similar reduction in pain scores with $300 \mathrm{mg}$ pregabalin administered twice before surgery. Singh et al in 2016 [10] also showed efficacy of 150 and $300 \mathrm{mg}$ pregabalin in decreasing pain scores. Peng et al in 2010 [11] showed that pain after laparoscopic cholecystectomy was significantly decreased with $75 \mathrm{mg}$ pregabalin but not with $50 \mathrm{mg}$ pregabalin. 
Contradictory results have been seen in a study by Chang et al in 2009 [12]. They found that perioperative use of pregabalin in two doses of $150 \mathrm{mg} 1$ hour before surgery and then 12 hours after the first dose did not decrease the frequency or severity of shoulder pain as well as the severity of pain after laparoscopic cholecystectomy.

\subsection{Opioid Consumption}

In our study, we did not find a significant difference in opioid consumption between the placebo and pregabalin groups. This can be explained by the relatively modest reduction in pain scores between the groups. This is also because pain is a subjective feeling but requirement of analgesic is a hard end point and was negative in our study. This might be one of the reasons why in spite of multiple studies showing decrease in pain, it has not become a routine practice to use pre-emptive pregablin. One interesting finding was the very low requirement of rescue analgesia in our study in almost both the groups. This could be related to either surgical technique or the efficacy of intra-operative anesthesia. Similar to our study, Peng et al in 2010 [11] also had found that although 50 and $75 \mathrm{mg}$ oral pregablin were able to decrease pain scores, analgesic requirement in the form of fentanyl was not decreased. Chang et al [12] had also showed that pain scores, time for first rescue analgesia and additional need of ketolorac consumption did not differ significantly in pregabalin vs placebo. This is in contrast to other studies by Agarwal, Balaban, Sarakatsianou and Singh et al [7-10]. Bekawi et al had reported very different results than all previous studies [13]. In 2014, they had showed that $150 \mathrm{mg}$ pregabalin perioperatively is effective in reducing postoperative pethidine consumption without increasing the side effect profile, although it has failed to reduce postoperative pain (VAS scores).

\subsection{Side-Effects}

Pregablin arm showed similar incidence of nausea and vomiting compared to placebo. This may be explained on the association of greater VAS score in the control group with increased demand of rescue opoid analgesic. In a recent study by Singh [10], the incidence of side effects such as nausea and vomiting were minimal in the two pregabalin groups as compared with the control which recorded increase incidence, and the same had been reported by different independent studies of Paul FW et al [14], Wichai I et al [15] and Bekawi et al. [13] Similar to postoperative nausea and vomiting, we did not find a significant difference in dizziness and sedation between pregabalin or placebo arms. This translates into almost similar side effect profile with pregablin compared to placebo and shows the safety of this intervention in decreasing post operative pain in patients undergoing laparoscopic cholcystectomy. Balaban et al, Chang et al, Esmat et al and Girija et al reported increased postoperative sedation early (at $15 \mathrm{~min}$ and $2 \mathrm{~h}$, respectively) in the intervention group (300 mg PG) $[8,12,16,17]$. Sarakatsianou had reported an increase in incidence of dizziness which was significantly higher in the PG group during the first 8 hours postoperatively and occurred in $70 \%$ of patients [9]. Patients receiving pregabalin exhibited a significant extubation time delay. This variable has not been evaluated in previous studies, so it is not known if it is a constant consequence or represents a dose-related effect, as they had used a total of $600 \mathrm{mg}$ of pregablin.

\section{Conclusions}

Based on our study, we conclude that use of pre-emptive $150 \mathrm{mg}$ oral Pregabalin 1 hour before surgery is a safe and effective method for post-operative analgesia in patients undergoing laparoscopic cholecystectomy. Further studies are needed to study patient satisfaction, time taken to return to work and length of hospital stay.

\section{References}

[1]. Wilson RG, Macintyre IM, Nixon SJ, Saunders JH, Varma JS, King PM. Laparoscopic cholecystectomy as a safe and effective treatment for severe acute cholecystitis. BMJ (Clinical research ed). 1992 Aug;305(6850):394(6).

[2]. Wills VL, Hunt DR. Pain after laparoscopic cholecystectomy. The British journal of surgery. 2000;87(3):273 \{84\}.

[3]. Weinbroum AA. Non-opioid IV adjuvants in the perioperative period: pharmacological and clinical aspects of ketamine and gabapentinoids. Pharmacological research : the official journal of the Italian Pharmacological Society. 2012;65(4):411\{29\}.

[4]. Ben Menachem E. Pregabalin pharmacology and its relevance to clinical practice. Epilepsia. 2004 Aug 1;45(s6):13-8.

[5]. Mitra S, Khandelwal P, Roberts K, Kumar S, Vadivelu N. Pain relief in laparoscopic cholecystectomy\{a review of the current options. Pain practice: the official journal of World Institute of Pain. 2012;12(6):485\{96\}

[6]. Gurusamy KS, Vaughan J, Toon CD, et al.Pharmacological interventions for prevention or treatment of postoperative pain in people undergoing laparoscopic cholecystectomy. 2014 The Cochrane Collaboration, Published by John Wiley \& Sons, Ltd. 2014

[7]. Agarwal A, Gautam S, Gupta D, et al. Evaluation of a single preoperative dose of pregabalin for attenuation of postoperative pain after laparoscopic cholecystectomy. Br JAnaesh 2008;101:700-4.

[8]. BalabanF, YağarS, ÖzgökA et al. A randomized, placebo-controlled study of pregabalin for postoperative pain intensity after laparoscopic cholecystectomy. J ClinAnesth 2012;24:175-8.

[9]. Sarakatsianou C, Theodorou E, Georgopoulou S, et al. Effect of pre-emptive pregabalin on pain intensity and postoperative morphine consumption after laparoscopic cholecystectomy. SurgEndosc 2013; 27(7):2504-11.

[10]. Singh et al. Pregabalin for Post-Cholecystectomy Pain Relief- A Study on the Response of Two Different Doses. International Journal of Health Sciences \& Research. Vol.4; Issue: 5; May 2014 
[11]. Peng P.W.H, Li C, Farcas E, et al.Use of low-dose pregabalin in patients undergoing laparoscopic cholecystectomy. Br J Anaesth2010;105(2):155-61.

[12]. Chang SH, Lee HW, Kim SH, et al. An Evaluation of Perioperative Pregabalin for Prevention and Attenuation of Postoperative Shoulder Pain After Laparoscopic Cholecystectomy. AnesthAnalg 2009; 109:1284-6.

[13]. Bekawi MS, El Wakeel LM, Al Taher WM, et al. Clinical study evaluating pregabalin efficacy and tolerability for pain management in patients undergoing laparoscopic cholecystectomy. Clin J Pain 2014; 30(11):944-52.

[14]. Paul FW, Burcu T, Jimmie T, Kevin K. The effects of pregabalin on preoperative anxiety and sedation levels: a dose ranging study. Anaesth Analg 2009; 108(4): 1440-45.

[15]. Wichai I, Tanit V, Mutita K, Wachirapol K, Pipat P, Suyawej R. Effects of pregabalin on postoperative morphine consumption after abdominal hysterectomy with/without salphingo-oophorectomy: a randomized, double-blind trial. Journal of medical Assoc Thai 2009; 92(10): 1318-23

[16]. Esmat I, Farag H. Comparative study between paracetamol and two different doses of pregabalin on postoperative pain in laparoscopic cholecystectomy. Saudi Journal of Anesthesia. Vol. 9, Issue 4, October-December 2015.

[17]. Girija PR, Rahul Y, Arvind C, Hari HD.A63 Effects of pregabalin on postoperative pain and preoperative anxiety in patients undergoing lumbar discectomy. Eur J Anaesth 2012, Vol 29 Supplement 49 Neuroanaesth S19. 